Bulletin of the Section of Logic

Volume 47/1 (2018), pp. 33-44

http://dx.doi.org/10.18778/0138-0680.47.1.03

S. Khosravi Shoar, R. A. Borzooei, R. Moradian, A. Radfar

\title{
PC-LATTICES: A CLASS OF BOUNDED BCK-ALGEBRAS
}

\begin{abstract}
In this paper, we define the notion of PC-lattice, as a generalization of finite positive implicative BCK-algebras with condition (S) and bounded commutative BCK-algebras. We investiate some results for Pc-lattices being a new class of BCK-lattices. Specially, we prove that any Boolean lattice is a PC-lattice and we show that if $X$ is a PC-lattice with condition $S$, then $X$ is an involutory BCK-algebra if and only if $X$ is a commutative BCK-algebra. Finally, we prove that any PC-lattice with condition $(\mathrm{S})$ is a distributive BCK-algebra.

Keywords: PC-lattice, BCK-lattice, Involutory BCK-algebras, Bounded commutative BCK-algebras

2010 Mathematics Subject Classification: 06F35, 03G25
\end{abstract}

\section{Introduction}

A BCK-algebra is an important class of logical algebras introduced by Y. Imai and K. Iséki in 1966 [8]. This notion originated from two different sources: one of them is based on the set theory, the other is from the classical and non-classical propositional calculi. Non-classical logic has become a considerable formal tool for computer science and artificial intelligence to deal with fuzzy information and uncertain information. In the first half of the nineteenth century, George Boole's attempts to formalize propositional logic led to the concept of Boolean algebras. Investigating the axiomatics of Boolean algebras at the end of the nineteenth century, Charles S. Peirce and Ernst Schröder found it useful to introduce the concept of a lattice. Dedekind also introduced modularity, a weakened form 
of distributivity. It was Garret Birkhoff's work in the mid-thirties that started the general development of lattice theory. In a brilliant series of papers he demonstrated the importance of lattice theory and showed that it provides a unifying framework for hitherto unrelated developments in many mathematical disciplines. In BCK-algebras some important lattices such as bounded commutative BCK-algebras, involutory BCK-algebras and bounded implicative BCK-algebras were defined and some of their important properties were proved $[7,12,14]$. In order to extend the theory of bounded BCK-algebras, we introduce the concept of PC-lattices and characterize their properties. We prove that the class of these lattices includes some currently known subclasses of BCK-lattices such as bounded commutative BCK-algebras(bounded commutative BCK-lattices), finite positive implicative BCK-algebras with condition $(\mathrm{S})$ and bounded implicative BCK-algebras (Boolean lattices). We study the relation between involutory BCK-algebras and PC-lattices, and show that in PC-lattices with condition (S), commutative BCK-algebras and involutory BCK-algebras coincide. Finally, we prove that any $\mathrm{PC}$-lattice with condition (S) is distributive and detect the relations between these BCK-lattices by a figure.

\section{Preliminaries}

In this section, we give some definations and theorems that will be used in the next sections.

Definition 2.1. [11] Let $\mathrm{X}$ be a set with a binary operation $*$ and a constant 0 . Then $(X, *, 0)$ is called a BCK-algebra if it satisfies the following axioms:

(BCK-1) $((x * y) *(x * z)) *(z * y)=0$,

$(\mathrm{BCK}-2) \quad(x *(x * y)) * y=0$,

(BCK-3) $x * x=0$,

(BCK-4) $x * y=0$ and $y * x=0$ imply $x=y$,

(BCK-5) $0 * x=0$.

for all $x, y, z \in X$. A partial order $\leq$ on $X$ can be defined by $x \leq y$ if and only if $x * y=0$.

Proposition 2.2. [11] In any BCK-algebra X, the following hold:

(i) $(x * y) * z=(x * z) * y$,

(ii) $x \leq y$ implies $x * z \leq y * z$ and $z * y \leq z * x$, 
(iii) $x * y \leq x$,

(iv) $x * y \leq z \Leftrightarrow x * z \leq y$.

for all $x, y, z \in X$.

Definition 2.3. $[1,11,13,12]$ Let $\mathrm{X}$ be a BCK-algebra. Then, for all $x, y, z \in X$ :

(i) $X$ is called a positive implicative BCK-algebra if $(x * y) * z=(x * z) *(y * z)$,

(ii) $X$ is called an implicative BCK-algebra if $x *(y * x)=x$,

(iii) $X$ is called a commutative BCK-algebra if $x *(x * y)=y *(y * x)$,

(iv) $X$ is called bounded BCK-algebra, if there exists the greatest element 1 of $X$, and for any $x \in X, 1 * x$ is denoted by $N x$,

(v) $X$ is called involutory BCK-algebra, if for all $x \in X, N N x=x$.

Proposition 2.4. [11, 12] Let $X$ be a bounded BCK-algebra. Then, for all $x, y \in X$ :

(i) $N 1=0$ and $N 0=1$,

(ii) $N N x \leq x$ that $N N x=N(N x)$,

(iii) $N x * N y \leq y * x$,

(iv) $y \leq x$ implies $N x \leq N y$,

(v) $N x * y=N y * x$,

(vi) $N N N x=N x$.

TheOrem 2.5. [7] Let $X$ be a bounded BCK-algebra. Then for any $x, y \in$ $X$, the following hold:

(i) $X$ is involutory,

(ii) $x * y=N y * N x$,

(iii) $x * N y=y * N x$,

(iv) $x \leq N y$ implies $y \leq N x$.

THEOREM 2.6. [11] Every implicative BCK-algebra is a commutative and positive implicative BCK-algebra.

Definition 2.7. $[9,6]$ Let $X$ be a BCK-algebra. Then:

(i) $X$ is said to have condition (S) (S), if for any $x, y \in X$, the set $A(x, y)=$ $\{t \in X: t * x \leq y\}$ has the greatest element which is denoted by $x \circ y$,

(ii) $(X, *, \leq)$ is called a BCK-lattice, if $(X, \leq)$ is a lattice, where $\leq$ is the partial BCK-order on $X$, which has been introduced in Definition 2.1. 
Proposition 2.8. [9] Let $X$ be a BCK-algebras with condition (S). Then, for all $x, y, z \in X$ :

(i) $y \leq x \circ(y * x)$,

(ii) $(x \circ z) *(y \circ z) \leq x * y$,

(iii) $(x * y) * z=x *(y \circ z)$,

(iv) if $x \leq y$, then $x \circ z \leq y \circ z$,

(v) $z * x \leq y \Longleftrightarrow z \leq x \circ y$.

Theorem 2.9. [10] Let $X$ be a BCK-algebra with condition (S). Then, for all $x, y, z \in X$, the following are equivalent:

(i) $X$ is a positive implicative,

(ii) $x \leq y$ implies $x \circ y=y$,

(iii) $x \circ x=x$,

(iv) $(x \circ y) * z=(x * z) \circ(y * z)$,

(v) $x \circ y=x \circ(y * x)$.

Theorem 2.10. [6, 7, 14] Let $X$ be a BCK-algebra.

(i) If $X$ is a finite positive implicative BCK-algebra with condition (S), then $(X, \leq)$ is a distributive lattice,

(ii) If $X$ is a BCK-algebra with condition ( $S$ ), then $X$ is positive implicative if and only if $(X, \leq)$ is an upper semilattice with $x \vee y=x \circ y$, for any $x, y \in X$,

(iii) If $X$ is a bounded commutative BCK-algebra, then BCK-lattice $(X, \leq)$ is a distributive lattice, where $x \wedge y=y *(y * x)$ and $x \vee y=N(N x \wedge N y)$.

TheOrem 2.11. [7] Let $X$ be an involutory BCK-algebra. Then the following are equivalent:

(i) $(X, \leq)$ is a lower semilattice,

(ii) $(X, \leq)$ is an upper semilattice,

(iii) $(X, \leq)$ is a lattice.

Theorem 2.12. [12] Let $X$ be a bounded BCK-algebra. Then:

(i) every commutative BCK-algebra is an involutory BCK-algebra.

(ii) any implicative BCK-algebra is a Boolean lattice (a complemented distributive lattice). 
Theorem 2.13. [13, 14] Let $X$ be a BCK-algebra. Then, for all $x, y, z \in X$, the following are equivalent:

(i) $X$ is commutative,

(ii) $x * y=x *(y *(y * x))$,

(iii) $x *(x * y)=y *(y *(x *(x * y)))$,

(iv) $x \leq y$ implies $x=y *(y * x)$.

\section{PC-lattices}

In this scetion, we define the notion of PC-lattice in BCK-algebras, which is a new class of bounded BCK-algebras.

Definition 3.1. Let $(X, *, \leq)$ be a BCK-lattice. Then $(X, *, \leq)$ is called a PC-lattice, if

$$
(z * x) *(y * x)=z *(x \vee y) \text { for all } x, y, z \in X .
$$

Note. In what follows, we show that the class of PC-lattices includes the finite Positive implicative BCK-algebras with condition (S) and bounded Commutative BCK-algebras that are two important classes of BCK-lattices. Hence this class of BCK-lattices is called PC-lattice.

ExAmple 3.2. (i) Let $X=\{0, a, b, 1\}$ be a chain, where $0 \leq a \leq b \leq 1$, and the operation $*$ on $X$ is defined as follows:

\begin{tabular}{l|llll}
$*$ & 0 & $a$ & $b$ & 1 \\
\hline 0 & 0 & 0 & 0 & 0 \\
$a$ & $a$ & 0 & 0 & 0 \\
$b$ & $b$ & $b$ & 0 & 0 \\
1 & 1 & 1 & 1 & 0
\end{tabular}

It is easy to check that $(X, *, \leq)$ is a PC-lattice.

(ii) Let $X$ be an interval $[0,1]$ of real numbers and binary operation $*$ on $X$ is defined as a follows:

$$
x * y= \begin{cases}0 & \text { if } x \leq y \\ x & \text { if } x>y\end{cases}
$$

Then it is easy to check that $(X, * \leq)$ is a PC-lattice, where $x \wedge y=$ $\min \{x, y\}$ and $x \vee y=\max \{x, y\}$. 
The following example shows that a BCK-lattice is not a PC-lattice, in general.

EXAmple 3.3. Let $X=\{0, a, b, 1\}$ be a chain, that is $0 \leq a \leq b \leq 1$, and the operation $*$ on $X$ is defined as follows:

\begin{tabular}{c|cccc}
$*$ & 0 & $a$ & $b$ & 1 \\
\hline 0 & 0 & 0 & 0 & 0 \\
$a$ & $a$ & 0 & 0 & 0 \\
$b$ & $b$ & $b$ & 0 & 0 \\
1 & 1 & $b$ & $a$ & 0
\end{tabular}

Then it is easy to check that $(X, *, \leq)$ is a BCK-lattice but it is not a PC-lattice, since

$$
(1 * a) *(b * a)=b * b=0 \neq a=1 * b=1 *(b \vee a) .
$$

TheOrem 3.4. Let $X$ be a bounded BCK-algebra. Then:

(i) every commutative BCK-algebra is a PC-lattice.

(ii) every finite positive implicative BCK-algebra with condition $(S)$ is a PC-lattice

Proof: (i) Let $X$ be a commutative BCK-algebra. By Theorem 2.10 (iii), $X$ is a BCK-lattice and by Theorem 2.12 (i), $X$ is an involutory BCK-algebra. Hence for any $x, y, z \in X$, we have:

$$
\begin{aligned}
(z * x) *(y * x) & =(N x * N z) *(N x * N y) \text {, by Theorem 2.5 (ii) } \\
& =(N x *(N x * N y)) * N z \text {, by Proposition 2.2(i) } \\
& =(N x \wedge N y) * N z, \text { by Theorem } 2.10 \text { (iii) } \\
& =N N(N x \wedge N y) * N z \text {, by Definition } 2.3(\mathrm{v}) \\
& =z * N(N x \wedge N y), \text { by Theorem } 2.5 \text { (ii) } \\
& =z *(x \vee y) . \text { by Theorem } 2.10 \text { (iii) }
\end{aligned}
$$

Therefore, $X$ is a PC-lattice.

(ii) Let $X$ be a finite positive implicative BCK-algebra with condition $(S)$. Then by Theorem 2.10 (i), $X$ is a BCK-lattice and so for any $x, y, z \in X$,

$$
\begin{aligned}
(z * x) *(y * x) & =z *(x \circ(y * x)), \text { by Proposition } 2.8 \text { (iii) } \\
& =z *(x \circ y), \text { by Theorem } 2.9(\mathrm{v}) \\
& =z *(x \vee y) . \text { by Theorem } 2.10 \text { (ii) }
\end{aligned}
$$

Hence $X$ is a PC-lattice. 
The following example shows that the converse of Theorem 3.4 does not hold, in general.

ExAmple 3.5. Let $X=\{0, a, b, 1\}$ be a chain, where $0 \leq a \leq b \leq 1$, and the operation $*$ on $X$ is defined as follows:

\begin{tabular}{c|cccc}
$*$ & 0 & $a$ & $b$ & 1 \\
\hline 0 & 0 & 0 & 0 & 0 \\
$a$ & $a$ & 0 & 0 & 0 \\
$b$ & $b$ & $a$ & 0 & 0 \\
1 & 1 & 1 & 1 & 0
\end{tabular}

It is easy to check that $X$ is a PC-lattice, but it is not a positive implicative BCK-algebra, since $(b * a) *(a * a)=a * 0=a \neq 0=a * a=(b * a) * a$. Also $X$ is not a commutative BCK-algebra, since $1 *(1 * a)=1 * 1=0 \neq$ $a=a * 0=a *(a * 1)$.

Corollary 3.6. Any bounded implicative BCK-algebra (Boolean lattice) is a PC-lattice.

Proof: By Theorems 2.6, 2.12(i) and 3.4(i), the proof is clear.

Proposition 3.7. Let $X$ be a $P C$-lattice. Then for all $x, y, z \in X$ :

$$
(x \vee y) * z=(x * z) \vee(y * z)
$$

Proof: Suppose that $\mathrm{X}$ is a PC-lattice, and $x, y, z \in X$. Since $x \leq x \vee y$ and $y \leq x \vee y$, by Proposition 2.2 (ii), we have $(x * z) \leq(x \vee y) * z$ and $(y * z) \leq(x \vee y) * z$. Therefore, $(x * z) \vee(y * z) \leq(x \vee y) * z$. On the other hand, since $X$ is a PC-lattice, by Definition 3.1 used repeatedly, we have:

$$
\begin{aligned}
{[(x \vee y) * z] *[(x * z) \vee(y * z)] } & =[((x \vee y) * z) *(x * z)] *[(y * z) *(x * z)] \\
& =((x \vee y) *(x \vee z)) *((y * z) *(x * z)) \\
& =((x \vee y) *(x \vee z)) *(y *(x \vee z)) \\
& =(x \vee y) *((x \vee z) \vee y) \\
& =0
\end{aligned}
$$

Hence, $(x \vee y) * z \leq(x * z) \vee(y * z)$. Therefore $(x \vee y) * z=(x * z) \vee(y * z)$. 
TheOrem 3.8. Let $X$ be a bounded BCK-algebras.

(i) If $X$ is a finite PC-lattice, then $X$ is a BCK-algebra with condition (S). (ii) If $X$ is a BCK-algebra with condition (S), then $x \vee y=x \circ(y * x)$, for $x, y \in X$.

Proof: (i) Suppose that $(X, *, \leq)$ is a finite PC-lattice and $x, y \in X$. Then the set $A(x, y)=\{t: t * x \leq y\}$ is a finite subset of $X$. Let $A(x, y)=$ $\left\{t_{i} \mid i \in I\right\}$, where $I=\{1,2, . ., n\}$. Since $X$ is a lattice, there exists $z \in X$ such that $\bigvee\left\{t_{i}: t_{i} * x \leq y, i \in I\right\}=z$. Hence, by Proposition 3.7(ii),

$$
z * x=\left(\bigvee_{i \in I} t_{i}\right) * x=\bigvee_{i \in I}\left(t_{i} * x\right) \leq \bigvee y=y
$$

Therefore, $z \in A(x, y)$. It is clear that for every $w \in A(x, y), w \leq z$, and so $z$ is the greatest element of the set $A(x, y)$, which is defined by $x \circ y$. Hence every finite PC-lattice is a BCK-algebra with condition (S).

(ii) Suppose that $X$ is a PC-lattice with condition (S). Then for $x, y \in X$, the set $A(x, y * x)$ has the greatest element. Now, since

$$
((x \vee y) * x) *(y * x)=(x \vee y) *(x \vee y)=0
$$

we have $(x \vee y) * x \leq y * x$. Hence $x \vee y \in A(x, y * x)$. If $t \in A(x, y * x)$, then $t * x \leq y * x$, and so $(t * x) *(y * x)=0$. Since $\mathrm{X}$ is a PC-lattice, $t *(x \vee y)=(t * x) *(y * x)=0$ and so $t \leq x \vee y$.

Hence, $x \vee y$ is the greatest element of $A(x, y * x)$, that is $x \circ(y * x)=x \vee y$.

The following example shows that the converse of Theorem 3.8, is not correct in general.

Example 3.9. Let $X=\{0, a, b, 1\}$ be a chain, where $0 \leq a \leq b \leq 1$, and the operation $*$ on $X$ is defined as follows:

\begin{tabular}{c|cccc}
$*$ & 0 & $a$ & $b$ & 1 \\
\hline 0 & 0 & 0 & 0 & 0 \\
$a$ & $a$ & 0 & 0 & 0 \\
$b$ & $b$ & $a$ & 0 & 0 \\
1 & 1 & $a$ & $a$ & 0
\end{tabular}

\begin{tabular}{l|llll}
$\circ$ & 0 & $a$ & $b$ & 1 \\
\hline 0 & 0 & $a$ & $b$ & 1 \\
$a$ & $a$ & 1 & 1 & 1 \\
$b$ & $b$ & 1 & 1 & 1 \\
1 & 1 & 1 & 1 & 1
\end{tabular}

Then $X$ is a BCK-algebra with condition (S), but it is not a PC-lattice. Since

$$
(1 * a) *(b * a)=a * a=0 \neq a=1 * b=1 *(b \vee a)
$$

Also we have $a \circ(b * a)=a \circ a=1 \neq a \vee b=b$. 
Theorem 3.10. Let $X$ be a BCK-lattice. Then:

(i) $x *(y \wedge z)=(x * y) \vee(x * z)$, for $x, y, z \in X$,

(ii) if $X$ is with condition $(S)$, then for $x, y, z \in X, x \circ(y \wedge z)=(x \circ y) \wedge(x \circ z)$.

Proof: (i) Suppose that $X$ is a BCK-lattice and $x, y, z \in X$. Since $y \wedge z \leq$ $y$ and $y \wedge z \leq z$, by Proposition 2.2 (ii), we have $x * z \leq x *(y \wedge z)$ and $x * y \leq x *(y \wedge z)$. Hence

$$
(x * y) \vee(x * z) \leq x *(y \wedge z)
$$

Now it remains to prove that $x *(y \wedge z) \leq(x * y) \vee(x * z)$. Since $(x * y) \leq$ $(x * y) \vee(x * z)$, by Proposition 2.2(ii), and BCK-2, we have $x *((x * y) \vee$ $(x * z)) \leq x *(x * y) \leq y$, and similarly we have $x *((x * y) \vee(x * z)) \leq$ $x *(x * z) \leq z$. Hence $x *((x * y) \vee(x * z)) \leq y \wedge z$. By Proposition 2.2 (iv), we conclude that

$$
x *(y \wedge z) \leq(x * y) \vee(x * z)
$$

Therefore

$$
x *(y \wedge z)=(x * y) \vee(x * z)
$$

(ii) Suppose that $X$ is a BCK-lattice with condition (S) and $x, y, z \in X$. Since $y \wedge z \leq y$ and $y \wedge z \leq z$, by Proposition 2.8 (iv), we have $x \circ(y \wedge z) \leq$ $(x \circ y)$ and $x \circ(y \wedge z) \leq(x \circ z)$, and so

$$
x \circ(y \wedge z) \leq(x \circ y) \wedge(x \circ z)
$$

Now it remains to prove that $(x \circ y) \wedge(x \circ z) \leq x \circ(y \wedge z)$. Since $(x \circ y) \wedge(x \circ z) \leq$ $x \circ y$, by Proposition 2.8(v), we have $((x \circ y) \wedge(x \circ z)) * x \leq y$. In the same way we have $((x \circ y) \wedge(x \circ z)) * x \leq z$. Hence $((x \circ y) \wedge(x \circ z)) * x \leq y \wedge z$, and so

$$
(x \circ y) \wedge(x \circ z) \leq x \circ(y \wedge z)
$$

Therefore

$$
(x \circ y) \wedge(x \circ z)=x \circ(y \wedge z)
$$

Theorem 3.11. Let $X$ be a PC-lattice with condition (S). Then the following are equivalent:

(i) $X$ is an involutory $B C K$-algebra,

(ii) $X$ is a commutative BCK-algebra. 
Proof: $(i) \Rightarrow($ ii $)$ Let $X$ be an involutory BCK-algebra and $y \leq x$. Since $N y * N y=0$ and $N x * N y=(1 * x) *(1 * y) \leq y * x=0$, it follows that $(N x * N y) \vee(N y * N y)=0$. Hence:

$$
\begin{aligned}
(N x \circ(x * y)) * N y & =(N x \circ(N y * N x)) * N y, \text { by Theorem 2.5(ii) } \\
& =(N x \vee N y) * N y, \text { by Theorem } 3.8 \\
& =(N x * N y) \vee(N y * N y), \text { by Proposition 3.7(ii) } \\
= & 0
\end{aligned}
$$

Hence,

$$
\begin{aligned}
y *(x *(x * y)) & =y *(N N x *(x * y)) \\
& =y *((1 * N x) *(x * y)) \\
& =y *(1 *(N x \circ(x * y))), \text { by Proposition 2.8(iii) } \\
& =N N y * N(N x \circ(x * y)) \\
\leq & (N x \circ(x * y)) * N y \\
& =0
\end{aligned}
$$

Therefore, $y \leq x *(x * y)$. On the other hand, by BCK-2, we have $x *(x * y) \leq$ $y$. Consequently, $y=x *(x * y)$. Hence by Theorem $2.13,(X, *, \leq)$ is a commutative BCK-algebra.

$($ ii $) \Rightarrow(i)$. The proof holds by Theorem $2.12(\mathrm{i})$.

Theorem 3.12. Let $X$ be a PC-lattice with condition (S). Then $X$ is a distributive lattice.

Proof: Suppose that $X$ is a PC-lattice with condition (S). It is clear that $x \vee(y \wedge z) \leq(x \vee y) \vee(x \vee z)$, for any $x, y, z \in X$. Now it remains to show that $x \vee(y \wedge z) \geq(x \vee y) \wedge(x \vee z)$. Since $X$ satisfies the condition (S), we have;

$$
\begin{aligned}
x \vee(y \wedge z)=(y \wedge z) \vee x= & (y \wedge z) \circ(x *(y \wedge z)), \text { by Theorem 3.8(ii) } \\
= & (x *(y \wedge z)) \circ(y \wedge z) \\
= & ((x *(y \wedge z)) \circ y) \wedge((x *(y \wedge z)) \circ z), \\
& \text { by Proposition 3.7(i) } \\
\geq & ((x * y) \circ y) \wedge((x * z) \circ z) \\
= & (x \vee y) \wedge(x \vee z), \text { by Theorem 3.8(ii) }
\end{aligned}
$$

Therefore, $X$ is a distributive lattice. 


\section{Conclusion}

It is well-known that the concept of the lattice theory has an important role in investigating the structure of a logical system. Also a Boolean lattice, that is a complemented distributive lattice, has many applications in the computer science. In order to extend the concept of BCK-algebras, we have proposed the concepts of PC-lattices. Then we have established the relationships between these lattices and other currently known lattices in bounded BCK-algebras and proved that PC-lattices are distributive.

In the following diagram we can see the relation between some kinds of BCK-lattices in bounded BCK-algebras.

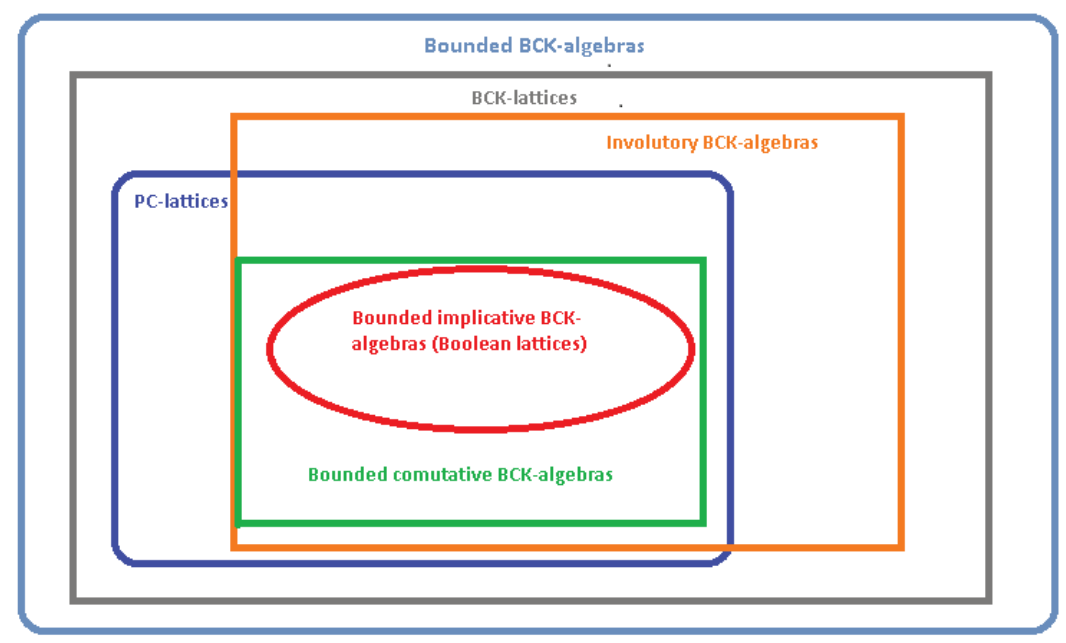

\section{Acknowledgments}

The authors would like to thank the referees for their valuable suggestions and comments. 


\section{References}

[1] C. Bărbăcioru, Positive implicative BCK-algebras, Mathematica Japonica 36 (1967), pp. 11-59.

[2] R. A. Borzooei, S. Khosravi Shoar, Implication Algebras are Equivalent to the Dual Implicative BCK-algebras, Scientiae Mathematicae Japonicae 633 (2006), pp. 429-431.

[3] R. A. Borzooei, S. Khosravi Shoar, R. Ameri, Some new filters in MTLalgebras, Fuzzy Sets and Systems 187(1) (2012), pp. 92-102.

[4] B. A. Davey, H. A. Priestley, Introduction to Lattices and Order, Camberidge University Press, 1990, 2002.

[5] G. Grätzer, General Lattice Theory, Academic Press, 1978.

[6] Y. Huang, BCI-algebras, Science Press, 2006.

[7] Y. Huang, On involutory BCK-algebras, Soochow Journal of Mathematics 32(1) (2006), pp. 51-57.

[8] Y. Imai, K. Iséki, On axioms systems of propositional calculi XIV, Proceedings of the Japan Academy 42 (1966), pp. 19-22.

[9] K. Iséki, BCK-algebras with condition (S), Mathematica Japonica 24 (1979), pp. 107-119.

[10] K. lséki, On positive implicative BCK-algebras with condition (S), Mathematica Japonica 24 (1979), pp. 107-119.

[11] K. Iséki and S. Tanaka, An introduction to the theory of BCK-algebras, Mathematica Japonica 23 (1978), pp. 1-26.

[12] J. Meng and Y. B. Jun, BCK-Algebras, Kyung Moon Sa Co, Seoul, Korea, 1994.

[13] S. Tanaka, A new class of algebras, Mathematics Seminar Notes 3 (1975), pp. 37-43.

[14] S. Tanaka, On ^-commutative algebras, Mathematics Seminar Notes 3 (1975), pp. 59-64.

Department of Mathematics, Fasa University, Fasa, Iran e-mail: khosravi.shoar@fasau.ac.ir

Department of Mathematics, Shahid Beheshti University, Tehran, Iran e-mail: borzooei@sbu.ac.ir

Department of mathematics Farhangian University, Tehran, Iran e-mail: Rmoradian2017@gmail.com

Payame Noor University, p. o. box. 19395-3697, Tehran, Iran e-mail: radfar@pnu.ac.ir 